\title{
On the exact distributional asymptotics for the supremum of a random walk with increments in a class of light-tailed distributions
}

\author{
Stan Zachary and Serguei Foss \\ Heriot-Watt University, Edinburgh \\ and \\ Institute of Mathematics, Novosibirsk
}

July 17, 2018

\begin{abstract}
We study the distribution of the maximum $M$ of a random walk whose increments have a distribution with negative mean and belonging, for some $\gamma>0$, to a subclass of the class $\mathcal{S}_{\gamma}$ - see, for example, Chover, Ney, and Wainger (1973). For this subclass we give a probabilistic derivation of the asymptotic tail distribution of $M$, and show that extreme values of $M$ are in general attained through some single large increment in the random walk near the beginning of its trajectory. We also give some results concerning the "spatially local" asymptotics of the distribution of $M$, the maximum of the stopped random walk for various stopping times, and various bounds.
\end{abstract}

\section{Introduction}

For any distribution function $F$ on $\mathbb{R}$ define the function $\varphi_{F}: \mathbb{R}_{+} \rightarrow \mathbb{R}_{+} \cup\{\infty\}$ by

$$
\varphi_{F}(\alpha):=\int_{-\infty}^{\infty} e^{\alpha x} d F(x)=\mathbf{E} e^{\alpha X}
$$

where the random variable $X$ has distribution function $F$. Denote also by $\bar{F}$ the tail distribution function given by $\bar{F}(x)=1-F(x)$. Let $F^{* n}$ denote the $n$-fold convolution of $F$ with itself.

As usual, we shall say that a distribution function $F$ on $\mathbb{R}$ belongs to the class $\mathcal{L}_{\gamma}, \gamma \geq 0$, if and only if

$$
\bar{F}(x)>0 \text { for all } x, \quad \lim _{x \rightarrow \infty} \frac{\bar{F}(x-k)}{\bar{F}(x)}=e^{\gamma k}, \quad \text { for all fixed } k .
$$

Note that the class $\mathcal{L}_{0}$ is the usual class of long-tailed distributions. As in that case, for $F \in \mathcal{L}_{\gamma}$, the convergence above is necessarily uniform for all $k$ in any compact interval. Further, if $F \in \mathcal{L}_{\gamma}$, then $\gamma$ is uniquely defined by $\gamma=\sup \left\{\alpha: \varphi_{F}(\alpha)<\infty\right\}$. In this case we shall find it convenient to write $\hat{\varphi}_{F}$ for $\varphi_{F}(\gamma)$, which may be either finite or infinite. Note also that if $F \in \mathcal{L}_{\gamma}$ and $\hat{\varphi}_{F}<\infty$, then

$$
\bar{F}(x)=o\left(e^{-\gamma x}\right), \quad \text { as } x \rightarrow \infty .
$$


A distribution function $F$ on $\mathbb{R}$ belongs to the class $\mathcal{S}_{\gamma}, \gamma \geq 0$, if and only if $F \in \mathcal{L}_{\gamma}$, $\hat{\varphi}_{F}<\infty$, and

$$
\overline{F^{* 2}}(x) \sim 2 \hat{\varphi}_{F} \bar{F}(x), \quad \text { as } x \rightarrow \infty
$$

(where, for any two positive functions $f_{1}, f_{2}$ on $\mathbb{R}$, we write $f_{1}(x) \sim f_{2}(x)$ as $x \rightarrow \infty$ if $f_{1}(x) / f_{2}(x) \rightarrow 1$ as $\left.x \rightarrow \infty\right)$. We remark that $\mathcal{S}_{\gamma}$ is sometimes defined first for distribution functions $F$ on $\mathbb{R}_{+}$, and the class then extended to $F$ on the whole real line by requiring $F \mathbf{I}_{\mathbb{R}_{+}} \in \mathcal{S}_{\gamma}$, where $\mathbf{I}_{\mathbb{R}_{+}}$is the indicator function of $\mathbb{R}_{+}$. However, this is unnecessary, essentially because of the condition $F \in \mathcal{L}_{\gamma}$.

Let $M$ be the maximum of a random walk whose increments have distribution function $F$ with negative mean. For $F$ belonging to the well-known class $\mathcal{S}_{0}$ of subexponential distributions, the asymptotic distribution of the tail of $M$ was given by Pakes (1975) and Veraverbeke (1977), see also the earlier result by Borovkov (1976) for the subclass of distributions with regularly varying tails. In this case extreme values of $M$ are in general attained through some single large increment in the random walk-see Zachary (2004). This is the "principle of a single big jump". Further, conditional on $M$ exceeding $x$, the probability that it does so within any given finite time tends to zero as $x$ tends to infinity. For $F \in \mathcal{S}_{\gamma}$ for some $\gamma>0$, it necessary to distinguish various subclasses of $\mathcal{S}_{\gamma}$. In the case where $\hat{\varphi}_{F}>1$, or where $\hat{\varphi}_{F}=1$ and $\varphi_{F}^{\prime}(\gamma)<\infty$, the asymptotic distribution of $M$ is as given by the classical Cramér-Lundberg theory; in particular trajectories leading to extreme values of $M$ are typically approximately linear prior to the time at which $M$ is attained. However, our interest in the present paper is primarily in that subclass of $\mathcal{S}_{\gamma}$ for which $\hat{\varphi}_{F}<1$. Here the asymptotic distribution of the tail of $M$ was given by Borovkov (1976, Chapter 4) for the case where the function $G$ given by $G(x)=e^{\gamma x} \bar{F}(x)$ is regularly varying. Further, for $G$ regularly varying, a considerably more general investigation of sample path behaviour associated with extreme values of $M$ was given by Borovkov and Borovkov (2004). The result of Borovkov was extended from the regularly varying to the general case by Veraverbeke (1977). Bertoin and Doney (1996) showed that there is a gap in Veraverbeke's proof and proposed their own corrected version. All these proofs are analytic. Our present aim is to derive in this case the asymptotic form of $\mathbf{P}(M>x)$, as $x \rightarrow \infty$, using arguments which are entirely probabilistic. We also give a number of corollaries and associated results, which in particular provide further insight into the behaviour of the sample paths leading to extreme values of $M$. These show that for the case $\gamma>0, \hat{\varphi}_{F}<1$, the principle of a single big jump again holds, i.e. extreme values of $M$ are in general again attained through some single large increment in the random walk. However, in this case such extreme values are likely to occur close to the start of this process - see Remark 4 below for a precise statement, and again Borovkov and Borovkov (2004) for more on the case where $G$ is regularly varying. We also present results for the (spatially) local asymptotics for $M$, the asymptotics for the maximum of the stopped random walk (for various stopping times), and we give some bounds.

Finally in this section we give some general properties of the class $\mathcal{S}_{\gamma}, \gamma \geq 0$, which will be required subsequently. For $F \in \mathcal{L}_{\gamma}, \gamma \geq 0$, with $\hat{\varphi}_{F}<\infty$, it is straightforward to show that (3) is equivalent to the requirement that

$$
\lim _{x \rightarrow \infty} \frac{1}{\bar{F}(x)} \int_{h(x)}^{x-h(x)} d F(y) \bar{F}(x-y)=0,
$$

for any positive function $h$ such that

$$
h(x) \leq x / 2 \text { for all } x, \quad \lim _{x \rightarrow \infty} h(x)=\infty .
$$


The class $\mathcal{S}_{\gamma}$ is thus indeed a natural generalisation of the class $\mathcal{S}_{0}$ of subexponential distributions (recall also that $\hat{\varphi}_{F}=1$ for $F \in \mathcal{S}_{0}$ ). As in the case $\gamma=0$, it is not easy to give an example of a distribution $F$ which belongs to $\mathcal{L}_{\gamma}$ with $\hat{\varphi}_{F}<\infty$, but not to $\mathcal{S}_{\gamma}$, and all such examples are more-or-less artificial.

It is clear that, for $\gamma \geq 0$, the class $\mathcal{S}_{\gamma}$ is closed under tail-equivalence (i.e., if $F \in \mathcal{S}_{\gamma}$ and $\bar{F}(x) \sim c \bar{G}(x)$ with $0<c<\infty$, then $\left.G \in \mathcal{S}_{\gamma}\right)$. More generally, we have the result given by Proposition 1 below, the proof of which follows from Lemma 5.1 of Pakes (2004) analogously to the proof of Lemma 5.2 of that paper.

Proposition 1. Suppose that $F \in \mathcal{S}_{\gamma}$ for some $\gamma \geq 0$. Suppose further that, for $i=$ $1, \ldots, n$, the distribution function $F_{i}$ is such that $\overline{F_{i}}(x) \sim c_{i} \bar{F}(x)$ as $x \rightarrow \infty$ for some $c_{i} \geq 0$ (where in the case $c_{i}=0$ we understand this to mean that $\overline{F_{i}}(x)=o(\bar{F}(x)$ ) as $x \rightarrow \infty)$. Then $\hat{\varphi}_{F_{i}}<\infty$ for all $i=1, \ldots, n$ and the convolution $F_{1} * \cdots * F_{n}$ satisfies

$$
\overline{F_{1} * \cdots * F_{n}}(x) \sim \prod_{i=1}^{n} \hat{\varphi}_{F_{i}} \sum_{i=1}^{n} \frac{c_{i}}{\hat{\varphi}_{F_{i}}} \bar{F}(x), \quad \text { as } x \rightarrow \infty,
$$

Further, if $\sum_{i=1}^{n} c_{i}>0$ then $F_{1} * \cdots * F_{n} \in \mathcal{S}_{\gamma}$.

In particular we have the generalisation of the property (3) above, that if $F \in \mathcal{S}_{\gamma}$, then, for all $n \geq 1$,

$$
\overline{F^{* n}}(x) \sim n \hat{\varphi}_{F}^{n-1} \bar{F}(x), \quad \text { as } x \rightarrow \infty .
$$

For future reference, we note that, for $\gamma \geq 0$, the class $\mathcal{S}_{\gamma}$ may be extended to include distributions $F$ with support on $\mathbb{R} \cup\{-\infty\}$, i.e. we may allow the possibility of strictly positive mass at $-\infty$. For $\gamma>0$, Proposition 1 remains unchanged. This is also true for $\gamma=0$, provided that here, for any $F \in \mathcal{S}_{0}$, we take $\hat{\varphi}_{F}=\bar{F}(-\infty)$.

\section{Random walks with negative drift}

Let $\left\{\xi_{n}\right\}_{n \geq 1}$ be independent identically distributed random variables with distribution function $F$ on $\mathbb{R}$. Let $S_{0}=0, S_{n}=\sum_{i=1}^{n} \xi_{i}$ for $n \geq 1$. Let $M_{n}=\max _{0 \leq i \leq n} S_{i}$ for $n \geq 0$ and let $M=\sup _{n \geq 0} S_{n}$.

Suppose that $F \in \mathcal{L}_{\gamma}$ for some $\gamma>0$ and that $\hat{\varphi}_{F}<1$. Since $\varphi_{F}(0)=1$ and $\varphi_{F}$ is convex on $[0, \gamma]$, it follows that $\varphi_{F}^{\prime}(0)<0$ and so $F$ has a negative mean, which, we note, may be $-\infty$. It thus follows that $\mathbf{P}(M<\infty)=1$. We are interested in the asymptotic distribution of $\mathbf{P}(M>x)$ as $x \rightarrow \infty$.

The following lemma gives a useful preliminary result.

Lemma 1. Suppose that $F \in \mathcal{L}_{\gamma}$ for some $\gamma>0$ and that $\hat{\varphi}_{F}<1$. Then

$$
1 \leq \mathbf{E} e^{\gamma M} \leq \frac{1}{1-\hat{\varphi}_{F}}
$$

and therefore

$$
\mathbf{P}(M>x)=o\left(e^{-\gamma x}\right), \quad \text { as } x \rightarrow \infty .
$$

Proof. Observe that

$$
1 \leq e^{\gamma M} \leq \sum_{n=0}^{\infty} e^{\gamma S_{n}} .
$$

The result (6) now follows by taking expectations and noting that $\mathbf{E} e^{\gamma S_{n}}=\hat{\varphi}_{F}^{n}$. 
For any stopping time $\sigma$ define, on the set $\sigma<\infty$,

$$
M^{\sigma}=\sup _{m \geq 0} S_{\sigma+m}-S_{\sigma} .
$$

(In particular, for any finite $n, M^{n}=\sup _{m \geq 0} S_{n+m}-S_{n}$.) For each $x \geq a \geq 0$, and each $n \geq 1$, define the event

$$
A_{n}^{a, x}=\left\{M_{n-1} \leq a, S_{n}>x\right\} .
$$

Note that for any $a$ and $x$ as above, the events $A_{n}^{a, x}, n \geq 1$, are disjoint. The following lemma gives a lower bound.

Lemma 2. Suppose that $F \in \mathcal{L}_{\gamma}$ for some $\gamma>0$ and that $\hat{\varphi}_{F}<1$. Then, given $\varepsilon>0$, there exists $a>0$ such that

$$
\liminf _{x \rightarrow \infty} \frac{\mathbf{P}(M>x)}{\bar{F}(x)} \geq \liminf _{x \rightarrow \infty} \frac{1}{\bar{F}(x)} \mathbf{P}\left(M>x, \bigcup_{n \geq 1} A_{n}^{a, x-a}\right) \geq(1-\varepsilon) \frac{\mathbf{E} e^{\gamma M}}{1-\hat{\varphi}_{F}} .
$$

Proof. Observe first that, from the definition (11) and the monotonicity of $\bar{F}$, it follows straightforwardly that, given any $\gamma^{\prime} \in(0, \gamma)$, there exists $x_{0}$ such that, for all $x \geq x_{0}$ and all $t \leq-1$,

$$
\begin{aligned}
\bar{F}(x-t) & \leq \bar{F}(x) e^{\gamma t} e^{\left(\gamma^{\prime}-\gamma\right) t} \\
& =\bar{F}(x) e^{\gamma^{\prime} t} .
\end{aligned}
$$

We thus have, for any $a \in \mathbb{R}$ and any $n \geq 1$,

$$
\begin{aligned}
\mathbf{P}\left(S_{n-1} \leq a, S_{n}>x\right) & =\int_{-\infty}^{a} \mathbf{P}\left(S_{n-1} \in d t\right) \bar{F}(x-t) \\
& =(1+o(1)) \bar{F}(x) \int_{-\infty}^{a} \mathbf{P}\left(S_{n-1} \in d t\right) e^{\gamma t}
\end{aligned}
$$

as $x \rightarrow \infty$. This follows from (11) and the dominated convergence theorem, where (17) and (11), with the asserted uniformity, are used to bound the integrand respectively in the regions $(-\infty,-1)$ and $[-1, a]$.

Now fix $a>0$. For each $n \geq 1$, and for $x \geq a$,

$$
\begin{aligned}
\mathbf{P}\left(A_{n}^{a, x}\right) & =\mathbf{P}\left(S_{n-1} \leq a, S_{n}>x\right)-\mathbf{P}\left(M_{n-2}>a, S_{n-1} \leq a, S_{n}>x\right) \\
& \geq \mathbf{P}\left(S_{n-1} \leq a, S_{n}>x\right)-\mathbf{P}\left(M_{n-2}>a\right) \bar{F}(x-a) \\
& \geq \mathbf{P}\left(S_{n-1} \leq a, S_{n}>x\right)-\mathbf{P}(M>a) \bar{F}(x-a) \\
& =(1+o(1)) \lambda(n, a) \bar{F}(x)
\end{aligned}
$$

as $x \rightarrow \infty$, from (11) and (9), where

$$
\lambda(n, a)=\int_{-\infty}^{a} \mathbf{P}\left(S_{n-1} \in d t\right) e^{\gamma t}-\mathbf{P}(M>a) e^{\lambda a} .
$$

For any $n \geq 1, x \geq 2 a$,

$$
\begin{aligned}
\mathbf{P}\left(M>x, A_{n}^{a, x-a}\right) & \geq \int_{0}^{a} \mathbf{P}\left(A_{n}^{a, x-t}, M^{n} \in d t\right) \\
& =\int_{0}^{a} \mathbf{P}\left(A_{n}^{a, x-t}\right) \mathbf{P}(M \in d t) \\
& \geq(1+o(1)) \lambda(n, a) \int_{0}^{a} \mathbf{P}(M \in d t) \bar{F}(x-t) \\
& =(1+o(1)) \lambda(n, a) \bar{F}(x) \int_{0}^{a} \mathbf{P}(M \in d t) e^{\gamma t}
\end{aligned}
$$


as $x \rightarrow \infty$, where (12) follows since, for each $t$, the event $A_{n}^{a, x-t}$ and the random variable $M^{n}$ are independent and the latter is equal in distribution to $M$, and where (13) follows from (10). Since also the events $A_{n}^{a, x-a}, n \geq 1$, are disjoint, we have

$$
\liminf _{x \rightarrow \infty} \frac{1}{\bar{F}(x)} \mathbf{P}\left(M>x, \bigcup_{n \geq 1} A_{n}^{a, x-a}\right) \geq \sum_{n=1}^{N} \lambda(n, a) \int_{0}^{a} \mathbf{P}(M \in d t) e^{\gamma t}
$$

for all $N$. Recall that $\hat{\varphi}_{F}<1$. From (11), the definition of $\hat{\varphi}_{F}$, and Lemma 1 we see that

$$
\lim _{N \rightarrow \infty} \lim _{a \rightarrow \infty} \sum_{n=1}^{N} \lambda(n, a)=\sum_{n \geq 1} \hat{\varphi}_{F}^{n-1}=\frac{1}{1-\hat{\varphi}_{F}},
$$

while, also $\lim _{a \rightarrow \infty} \int_{0}^{a} \mathbf{P}(M \in d t) e^{\gamma t}=\mathbf{E} e^{\gamma M}$, so that the required result now follows from (14).

We now give the companion upper bound, which requires the stronger condition that $F \in \mathcal{S}_{\gamma}$

Lemma 3. Suppose that $F \in \mathcal{S}_{\gamma}$ for some $\gamma>0$ and that $\hat{\varphi}_{F}<1$. Then

$$
\limsup _{x \rightarrow \infty} \frac{\mathbf{P}(M>x)}{\bar{F}(x)} \leq \frac{\mathbf{E} e^{\gamma M}}{1-\hat{\varphi}_{F}} .
$$

Proof. For any sequence of events $\left\{E_{n}\right\}$ we make the convention: $\min \left\{n \geq 1: \mathbf{I}\left(E_{n}\right)=\right.$ $1\}=\infty$ if $\mathbf{I}\left(E_{n}\right)=0$ for all $n$. Since $\hat{\varphi}_{F}<1$, the distribution $F$ has a negative mean (which, as previously remarked, may be $-\infty$ ). Hence there exists $c<0$ such that

$$
\limsup _{n \rightarrow \infty} S_{n} / n<c \quad \text { a.s. }
$$

Given also $R>0$, define renewal times $0 \equiv \tau_{0}<\tau_{1} \leq \tau_{2} \leq \ldots$ for the process $\left\{S_{n}\right\}$ by

$$
\tau_{1}=\min \left\{n \geq 1: S_{n}>R+n c\right\} \leq \infty
$$

and, for $m \geq 2$,

$$
\begin{aligned}
\tau_{m} & =\infty, \quad \text { if } \tau_{m-1}=\infty, \\
\tau_{m} & =\tau_{m-1}+\min \left\{n \geq 1: S_{\tau_{m-1}+n}-S_{\tau_{m-1}}>R+n c\right\}, \quad \text { if } \tau_{m-1}<\infty .
\end{aligned}
$$

Observe that, for each $m \geq 1$, conditional on the event $\tau_{m-1}<\infty$, the distribution of $\left(\tau_{m}-\tau_{m-1}, S_{\tau_{m}}-S_{\tau_{m-1}}\right)$ is otherwise independent of $\mathcal{F}_{\tau_{m-1}}$ (where for each $n \geq 1$ the $\sigma$-algebra $\mathcal{F}_{n}$ is that generated by the process $\left.\left\{S_{k}\right\}_{k \leq n}\right)$ and is equal to that of $\left(\tau_{1}, S_{\tau_{1}}\right)$. In particular $\mathbf{P}\left(\tau_{m}<\infty\right)=\delta^{m}$ where

$$
\delta \equiv \mathbf{P}\left(\tau_{1}<\infty\right) \rightarrow 0, \quad \text { as } R \rightarrow \infty
$$

from (16). Define also $S_{\infty}=-\infty$.

Since the conditions of Lemma 2 are also satisfied here, it follows from (9) that, for any $n \geq 1$ and any $a$,

$$
\mathbf{P}\left(S_{n-1} \leq a, S_{n}>x\right) \leq(1+o(1)) \hat{\varphi}_{F}^{n-1} \bar{F}(x), \quad \text { as } x \rightarrow \infty .
$$


Fix also $\gamma^{\prime} \in(0, \gamma)$ and note that, from (7) and (8), for any $n \geq 1$ and any $a \leq-1$,

$$
\begin{aligned}
\mathbf{P}\left(S_{n-1} \leq a, S_{n}>x\right) & \leq(1+o(1)) \bar{F}(x) \int_{-\infty}^{a} \mathbf{P}\left(S_{n-1} \in d t\right) e^{\gamma^{\prime} t} \\
& \leq(1+o(1)) e^{\gamma^{\prime} a} \bar{F}(x) \quad \text { as } x \rightarrow \infty,
\end{aligned}
$$

uniformly in $n$ and in $a \leq-1$.

It follows from (18) and (19) that, for any $N$ such that $R+N c \leq-1$, as $x \rightarrow \infty$,

$$
\begin{aligned}
\mathbf{P}\left(S_{\tau_{1}}>x\right) & =\sum_{n \geq 1} \mathbf{P}\left(\tau_{1}=n, S_{n}>x\right) \\
& \leq \sum_{n \geq 1} \mathbf{P}\left(S_{n-1} \leq R+(n-1) c, S_{n}>x\right) \\
& \leq(1+o(1)) \bar{F}(x)\left(\sum_{n=1}^{N} \hat{\varphi}_{F}^{n-1}+\sum_{n>N} e^{\gamma^{\prime}(R+(n-1) c)}\right) .
\end{aligned}
$$

Since $c<0$, letting $N \rightarrow \infty$, we obtain

$$
\mathbf{P}\left(S_{\tau_{1}}>x\right) \leq(1+o(1)) \frac{\bar{F}(x)}{1-\hat{\varphi}_{F}} \quad \text { as } x \rightarrow \infty .
$$

We now show that, for sufficiently large $R$ and $x, \sum_{m \geq 1} \mathbf{P}\left(S_{\tau_{m}}>x\right)$ is comparable to $\mathbf{P}\left(S_{\tau_{1}}>x\right)$. Define $\Phi_{R}=\mathbf{E} e^{\gamma S_{\tau_{1}}}$. Then

$$
\begin{aligned}
\Phi_{R} & =\sum_{n \geq 1} \mathbf{E}\left(\mathbf{I}_{\left\{\tau_{1}=n\right\}} e^{\gamma S_{n}}\right) \\
& \leq \sum_{n \geq 1} \mathbf{E}\left(\mathbf{I}_{\left\{S_{n}>R+n c\right\}} e^{\gamma S_{n}}\right) \\
& \rightarrow 0 \quad \text { as } R \rightarrow \infty,
\end{aligned}
$$

by the dominated convergence theorem, since $\hat{\varphi}_{F}<1$ and, for each $n$,

$$
\mathbf{E}\left(\mathbf{I}_{\left\{S_{n}>R+n c\right\}} e^{\gamma S_{n}}\right) \leq \mathbf{E} e^{\gamma S_{n}}=\hat{\varphi}_{F}^{n} .
$$

For each $m \geq 1$, the distribution of the random variable $S_{\tau_{m}}$ is the $m$-fold convolution of the distribution of $S_{\tau_{1}}$ and belongs to $\mathcal{S}_{\gamma}$. Hence, from Proposition 1 (which, as already noted, extends to distributions in $\mathcal{S}_{\gamma}$ with positive mass at $-\infty$ ),

$$
\mathbf{P}\left(S_{\tau_{m}}>x\right)=(1+o(1)) m \Phi_{R}^{m-1} \mathbf{P}\left(S_{\tau_{1}}>x\right), \quad \text { as } x \rightarrow \infty
$$

We wish to use again the dominated convergence theorem to obtain the corresponding asymptotic result for $\sum_{m \geq 1} \mathbf{P}\left(S_{\tau_{m}}>x\right)$. To do so we work for the moment with conditional distributions. The distribution of $S_{\tau_{m}}$ conditional on $\tau_{m}<\infty$ is the $m$-fold convolution of the distribution of $S_{\tau_{1}}$ conditional on $\tau_{1}<\infty$. Further

$$
\mathbf{E}\left(e^{\gamma S_{\tau_{1}}} \mid \tau_{1}<\infty\right)=\delta^{-1} \Phi_{R}
$$

(where $\delta$ is as given by (17)). It therefore follows from Lemma 5.3 of Pakes (2004) that, given $\varepsilon>0$, there exists a constant $K$ such that, for all $m \geq 1$ and all $x \geq 0$,

$$
\begin{aligned}
\mathbf{P}\left(S_{\tau_{m}}>x\right) & =\delta^{m} \mathbf{P}\left(S_{\tau_{m}}>x \mid \tau_{m}<\infty\right) \\
& \leq \delta^{m} K\left(\max \left(1, \delta^{-1} \Phi_{R}+\varepsilon\right)\right)^{m} \mathbf{P}\left(S_{\tau_{1}}>x \mid \tau_{1}<\infty\right) \\
& =\delta^{-1} K\left(\max \left(\delta, \Phi_{R}+\delta \varepsilon\right)\right)^{m} \mathbf{P}\left(S_{\tau_{1}}>x\right)
\end{aligned}
$$


It now follows from (22) that, for sufficiently large $R$, we may use the dominated convergence theorem as required to obtain, from (23) and then (21),

$$
\begin{aligned}
\sum_{m \geq 1} \mathbf{P}\left(S_{\tau_{m}}>x\right) & =(1+o(1)) \mathbf{P}\left(S_{\tau_{1}}>x\right) \sum_{m \geq 1} m \Phi_{R}^{m-1}, \quad \text { as } x \rightarrow \infty \\
& \leq \frac{(1+o(1))}{1-\hat{\varphi}_{F}} \bar{F}(x) \sum_{m \geq 1} m \Phi_{R}^{m-1}, \quad \text { as } x \rightarrow \infty
\end{aligned}
$$

Since the random walk $\left\{S_{n}\right\}_{n \geq 0}$ attains its maximum $M$ almost surely, for any such sample path, the numbers $n=\min \left\{k: S_{k}=M\right\}$ and $m=\max \left\{i: \tau_{i} \leq n\right\}$ are finite. Then $M=S_{\tau_{m}}+M^{\tau_{m}}$ and $M_{\tau_{m}}<R$. Hence, for $x \geq 0$,

$$
\begin{aligned}
\mathbf{P}(M>x) & \leq \sum_{m \geq 1} \mathbf{P}\left(S_{\tau_{m}}+M^{\tau_{m}}>x, M^{\tau_{m}} \in[0, R]\right) \\
& =\sum_{m \geq 1} \int_{0}^{R} \mathbf{P}\left(M^{\tau_{m}} \in d t, S_{\tau_{m}}>x-t\right) \\
& =\int_{0}^{R} \mathbf{P}(M \in d t) \sum_{m \geq 1} \mathbf{P}\left(S_{\tau_{m}}>x-t\right) \\
& \leq \frac{(1+o(1))}{1-\hat{\varphi}_{F}} \sum_{m \geq 1} m \Phi_{R}^{m-1} \int_{0}^{R} \mathbf{P}(M \in d t) \bar{F}(x-t) \\
& =\frac{(1+o(1))}{1-\hat{\varphi}_{F}} \bar{F}(x) \sum_{m \geq 1} m \Phi_{R}^{m-1} \int_{0}^{R} e^{\gamma t} \mathbf{P}(M \in d t) \\
& \leq \frac{(1+o(1)) \mathbf{E} e^{\gamma M} \bar{F}(x) \sum_{m \geq 1} m \Phi_{R}^{m-1},}{1-\hat{\varphi}_{F}}
\end{aligned}
$$

where (25) follows since, conditional on $\tau_{m}<\infty$, the random variable $M^{\tau_{m}}$ is independent of $S_{\tau_{m}}$ and equal in distribution to $M$, the inequality (26) follows from (24) (since the integral is taken over the finite interval $[0, R]$ ), and the equation (27) follows from (11). Now let $R \rightarrow \infty$ in (28) and use (22) to obtain the required result.

By combining Lemmas 2 and 3, we obtain Theorem 11, where the final equality is given by letting $\varepsilon \rightarrow 0$.

Theorem 1. Suppose that $F \in \mathcal{S}_{\gamma}$ for some $\gamma>0$ and that $\hat{\varphi}_{F}<1$. Then, given $\varepsilon>0$, there exists $a>0$ such that

$$
(1-\varepsilon) \frac{\mathbf{E} e^{\gamma M}}{1-\hat{\varphi}_{F}} \leq \liminf _{x \rightarrow \infty} \frac{1}{\bar{F}(x)} \mathbf{P}\left(M>x, \bigcup_{n \geq 1} A_{n}^{a, x-a}\right) \leq \lim _{x \rightarrow \infty} \frac{\mathbf{P}(M>x)}{\bar{F}(x)}=\frac{\mathbf{E} e^{\gamma M}}{1-\hat{\varphi}_{F}} .
$$

Remark 1. It follows in particular from Theorem 1 that, given $\varepsilon>0$, there exists $a>0$ such that

$$
\liminf _{x \rightarrow \infty} \mathbf{P}\left(\bigcup_{n \geq 1} A_{n}^{a, x-a} \mid M>x\right)>1-\varepsilon,
$$

that is, for all sufficiently large $x$, conditional on the event $\{M>x\}$, at the time $\tau$ of the first jump of the process $\left\{S_{n}\right\}_{n \geq 0}$ above $a$, the value of $S_{\tau}$ exceeds $x-a$ with probability at least $1-\varepsilon$. This is the "principle of a single big jump". We note also that a more 
compact statement of the conclusion of the theorem is that, for any positive function $h$ satisfying the condition (5),

$$
\lim _{x \rightarrow \infty} \frac{1}{\bar{F}(x)} \mathbf{P}\left(M>x, \bigcup_{n \geq 1} A_{n}^{h(x), x-h(x)}\right)=\lim _{x \rightarrow \infty} \frac{\mathbf{P}(M>x)}{\bar{F}(x)}=\frac{\mathbf{E} e^{\gamma M}}{1-\hat{\varphi}_{F}} .
$$

Here again the events $A_{n}^{h(x), x-h(x)}, n \geq 1$, are disjoint, and we may replace the probability of a union of events by the appropriate sum of the individual probabilities.

Remark 2. While Theorem 1 does indeed give the asymptotic form of $\mathbf{P}(M>x)$ as $x \rightarrow \infty$, there seems to be no way to determine the constant $\mathbf{E} e^{\gamma M}$, which occurs in the statement of the theorem, in terms of the distribution function $F$. Hence the simple bounds given in (6) may be of use in applications.

We also give a "spatially local" result: from Theorem 11 and using again (1), we have immediately Theorem 2 below.

Theorem 2. Suppose that $F \in \mathcal{S}_{\gamma}$ for some $\gamma>0$ and that $\hat{\varphi}_{F}<1$. Then, for any fixed $t>0$, and for any positive function $h$ satisfying (15),

$\lim _{x \rightarrow \infty} \frac{1}{\bar{F}(x)} \mathbf{P}\left(M \in(x, x+t], \bigcup_{n \geq 1} A_{n}^{h(x), x-h(x)}\right)=\lim _{x \rightarrow \infty} \frac{\mathbf{P}(M \in(x, x+t])}{\bar{F}(x)}=\frac{\mathbf{E} e^{\gamma M}}{1-\hat{\varphi}_{F}}\left(1-e^{-\gamma t}\right)$,

with, for any $t_{0}>0$, uniformity in all $t \in\left[t_{0}, \infty\right]$.

Remark 3. The analogous result to Theorem 2 for the case $\gamma=0$ requires the slightly stronger condition that $F$ belong to the class $\mathcal{S}^{*}$ introduced by Klüppelberg (1988) - see Asmussen et al (2002) and Foss and Zachary (2004). However, for $\gamma>0$ the conditions analogous to those defining $\mathcal{S}_{0}$ and $\mathcal{S}^{*}$ match (see also the comments by Rogozin and Sgibnev (1999)).

Analogously to Theorem 11 we also have the following result for the maximum of the random walk on a finite time horizon.

Theorem 3. Suppose that $F \in \mathcal{S}_{\gamma}$ for some $\gamma>0$ and that $\hat{\varphi}_{F}<1$. Then, for each $N \geq 1$, and for any positive function $h$ satisfying (5),

$$
\lim _{x \rightarrow \infty} \frac{1}{\bar{F}(x)} \mathbf{P}\left(M_{N}>x, \bigcup_{1 \leq n \leq N} A_{n}^{h(x), x-h(x)}\right)=\lim _{x \rightarrow \infty} \frac{\mathbf{P}\left(M_{N}>x\right)}{\bar{F}(x)}=\sum_{n=1}^{N} \hat{\varphi}_{F}^{n-1} \mathbf{E} e^{\gamma M_{N-n}},
$$

where the convergence is uniform over all $N \leq \infty$.

Proof. The proof of Theorem 3 is simply a matter of checking that the proofs of Lemmas 2 and 3 continue to hold (with some small and straightforward adjustments in the case of Lemma 3) when restricted to a finite time horizon. The asserted uniformity in $N$ follows from the fact that the terms of the form $\hat{\varphi}_{F}^{n-1}$ occurring in (15) and (20) tend to zero as $n \rightarrow \infty$, coupled with the use of a simple truncation argument.

Remark 4. Since $\mathbf{E} e^{\gamma M_{n}} \rightarrow \mathbf{E} e^{\gamma M}$ as $n \rightarrow \infty$, and since $\hat{\varphi}_{F}<1$, it follows from Theorems and 3 that, given $\varepsilon>0$, there exists $N \geq 1$ such that

$$
\lim _{x \rightarrow \infty} \mathbf{P}\left(M_{N}>x \mid M>x\right)>1-\varepsilon
$$

that is, for all sufficiently large $x$, conditional on the random walk ever exceeding $x$, it does so by time $N$ with probability at least $1-\varepsilon$. As remarked in the Introduction, this behaviour is very different from that for the case $F \in \mathcal{S}_{0}$. 
Remark 5. Theorem 3 also has a "spatially local" version, analogous to Theorem 2.

The results of Theorem 3 may also be extended to random time horizons (with uniformity in all stopping times $\sigma \geq 1$ a.s.) We give a result for any a.s. finite stopping time $\sigma$ such that $S_{\sigma} \leq 0$ a.s. Examples of such stopping times are

$$
\sigma_{1}=\min \left\{n \geq 1: S_{n}<0\right\} \quad \text { and } \quad \sigma_{2}=\min \left\{n>\sigma_{1}: S_{n}<S_{\sigma_{1}}\right\}
$$

For such a stopping time, let $\chi=-S_{\sigma} \geq 0$ and note that $\mathbf{P}(\chi>0)>0$ since $\mathbf{E} \xi_{1}<0$.

Theorem 4. Suppose that $F \in \mathcal{S}_{\gamma}$ for some $\gamma>0$ and that $\hat{\varphi}_{F}<1$. Let $\sigma \geq 1$ be an a.s. finite stopping time such that $S_{\sigma} \leq 0$ a.s. Then

$$
\lim _{x \rightarrow \infty} \frac{\mathbf{P}\left(M_{\sigma}>x\right)}{\bar{F}(x)}=\left(1-\mathbf{E} e^{-\gamma \chi}\right) \frac{\mathbf{E} e^{\gamma M}}{1-\hat{\varphi}_{F}} .
$$

Proof. Since $\sigma$ is a.s. finite and $S_{\sigma} \leq 0$ a.s., it follows from Denisov (2005) that

$$
\mathbf{P}\left(M_{\sigma}>x\right) \sim \mathbf{P}\left(M \in\left(x, x+\chi^{\prime}\right)\right), \quad \text { as } x \rightarrow \infty,
$$

where $\chi^{\prime}$ is a copy of $\chi$ which is independent of $M$. The result (30) then follows from Theorem 2 and the dominated convergence theorem.

For a further extension to any stopping time, one can use the approach developed by Foss et al (2005). Indeed it follows from Remark 4 that it is sufficient to consider stopping times which are bounded almost surely from above by a fixed finite number $N$. Other extensions are also possible, for example, to "spatially local" versions of these results.

\section{Acknowledgement}

We are very grateful to Academician A. A. Borovkov and to Dmitry Korshunov for helpful and fruitful comments, and to Yuebao Wang for pointing out a small difficulty in an earlier version of the paper. 


\section{References}

[1] Asmussen, S., Kalashnikov, V., Konstantinides, D., Klüppelberg, C. and Tsitiashvili, G. (2002). A local limit theorem for random walk maxima with heavy tails. Statist. Probab. Lett., 56, 399-404.

[2] Bertoin, J. and Doney, R. A. (1996). Some asymptotic results for transient random walks. Adv. Appl. Prob., 28, 207-226.

[3] Borovkov, A. A. (1976). Stochastic Processes in Queueing Theory. Springer, New York.

[4] Borovkov, A. A. and Borovkov, K. A. (2004). On probabilities of large deviations for random walks II: regular exponentially decaying distributions. Theory Probab. Appl., 49, 189-206

[5] Chover, J., Ney, P. and Wainger, S. (1973). Functions of probability measures. J. Anal. Math., 26, 255-302.

[6] Denisov, D. (2005). A Note on the Asymptotics for the Maximum on a Random Time Interval of a Random Walk Markov. Proc. Rel. Fields, 11, 165-169.

[7] Foss, S., Palmowski, Z. and Zachary, S. (2005). The probability of exceeding a high boundary on a random time interval for a heavy-tailed random walk. Ann. Appl. Prob., 15, 1936-57.

[8] Foss, S. and Zachary, S (2003). The maximum on a random time interval of a random walk with long-tailed increments and negative drift, Ann. Appl. Prob., 13, 37-53.

[9] Klüppelberg, C. (1988). Subexponential distributions and integrated tails. J. Appl. Prob., 35, 325-347.

[10] Pakes, A. (1975). On the tails of waiting time distributions. J. Appl. Prob., 7, 745-789.

[11] Pakes, A. (2004). Convolution equivalence and infinite divisibility. J. Appl. Prob., 41, 407-424.

[12] Rogozin, B. A. and Sgibnev, M. S. (1999). Strongly subexponential distributions and Banach algebras of measures. Siberian Math. J., 40, 963-971.

[13] Veraverbeke, N. (1977). Asymptotic behavior of Wiener-Hopf factors of a random walk. Stoch. Proc. Appl., 5, 27-37.

[14] Zachary, S. (2004). A note on Veraverbeke's theorem. Queueing Systems, 46, 9-14. 